\title{
Resonating modes of vibrating microcantilevers in liquid
}

\author{
Murali Krishna Ghatkesar, Thomas Braun, ${ }^{\text {a) }}$ Viola Barwich, Jean-Pierre Ramseyer, \\ Christoph Gerber, Martin Hegner, ${ }^{\text {b) }}$ and Hans Peter Lang ${ }^{\text {() }}$ \\ National Center of Competence for Research in Nanoscience, Institute of Physics, University of Basel, \\ 4056 Basel, Switzerland
}

(Received 15 August 2007; accepted 22 December 2007; published online 29 January 2008)

\begin{abstract}
A study of nanomechanical cantilevers vibrating at various resonating modes in liquid is presented. Resonant frequency spectrum with 16 well resolved flexural modes is obtained. The quality factor increased from 1 at mode 1 to 30 at mode 16. The theoretical estimate of eigenfrequency using the Elmer-Dreier model [F.-J. Elmer and M. Dreier, J. Appl. Phys. 81, 12 (1997)] and Sader's extended viscous model [C. A. Van Eysden and J. E. Sader, J. Appl. Phys. 101, 044908 (2007)] matched well with the experimental data. The apparent mass of the liquid comoved by the oscillating cantilevers decreased asymptotically with mode number. (C) 2008 American Institute of Physics.
\end{abstract}

[DOI: $10.1063 / 1.2838295$ ]

High sensitivity, portability, multiple target sensing, diverse applicability, and low cost are the driving factors for the development of microcantilever array sensors. This versatile tool has already found application in the field of genomics, ${ }^{1-3}$ proteomics, ${ }^{4,5}$ food engineering, ${ }^{6}$ and chemistry. ${ }^{7}$ Surface stress (static mode) and mass change (dynamic mode) are the important parameters of interest for microcantilever applied as a sensor. ${ }^{8}$ The focus of this article is on the dynamic mode. It is a challenging task to vibrate a microcantilever array in liquid environment. The operation requires efficient coupling of external excitation energy to the microcantilevers avoiding anomalous additional acoustic frequencies originating from the liquid chamber. It also needs a bubble free fluid flow system, optimally focussed parallel laser beams, and sufficient laser power to pass through the air-liquid-air interface for optical detection of cantilever motion. We have operated a microcantilever array in liquid by overcoming these difficulties. A clear and well resolved frequency spectrum with 16 resonant modes of vibration with their corresponding quality factors was obtained. We focused on the comparison of the experimentally obtained eigenfrequencies with those obtained from analytical expressions of different theoretical models.

An array of eight microcantilevers, each $500 \mu \mathrm{m}$ long, $100 \mu \mathrm{m}$ wide and $1 \mu \mathrm{m}$ thick, externally excited by a piezoelement beneath the chip base, was used for the measurements [Fig. 1(a)]. The laser beam deflection detection technique is adapted to monitor resonance frequencies of the eight individual microcantilevers placed in a tiny chamber volume of $6 \mu$ l. Liquid was continuously flown at a rate of $10 \mu \mathrm{l} / \mathrm{min}^{9}{ }^{9}$ A spectrum of the resonance peaks with corresponding phase information spanning 16 flexural modes until $1 \mathrm{MHz}$ is shown in Fig. 1(b). The phase values are wrapped between $180^{\circ}$ and $-180^{\circ}$. A phase change of $180^{\circ}$ across every peak with a steep slope around the peak position is clearly observed (eigenfrequency).

The experimental eigenfrequencies extracted from the phase spectra [Fig. 1(b)] are shown in Fig. 2(a) as red circles. The corresponding quality factors determined from the am-

\footnotetext{
${ }^{a)}$ Electronic mail: braun.thomas@mac.com.

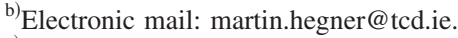

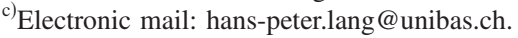

plitude peaks are displayed in Fig. 3(a). Note that the eigenfrequencies deviated by less than $1 \%$ among eight cantilevers in an array indicating their similar mechanical characteristics (see supplemental material). The quality factor increase from 1 for mode 1 to 30 for mode 16 indicates that the damping effect from surrounding medium decreases with mode number. The difference between the two adjacent

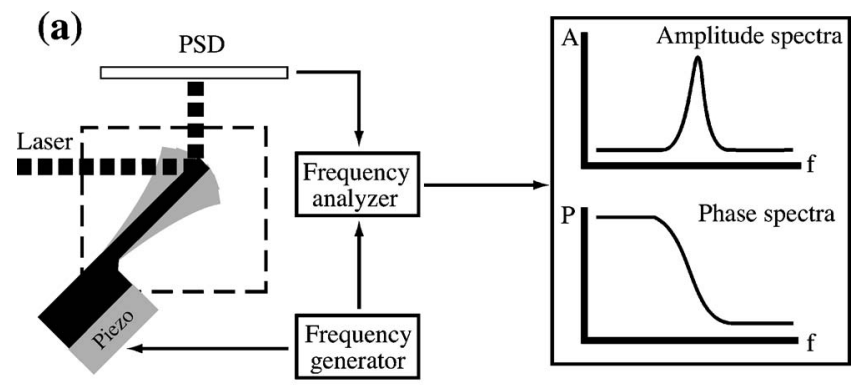

(b)

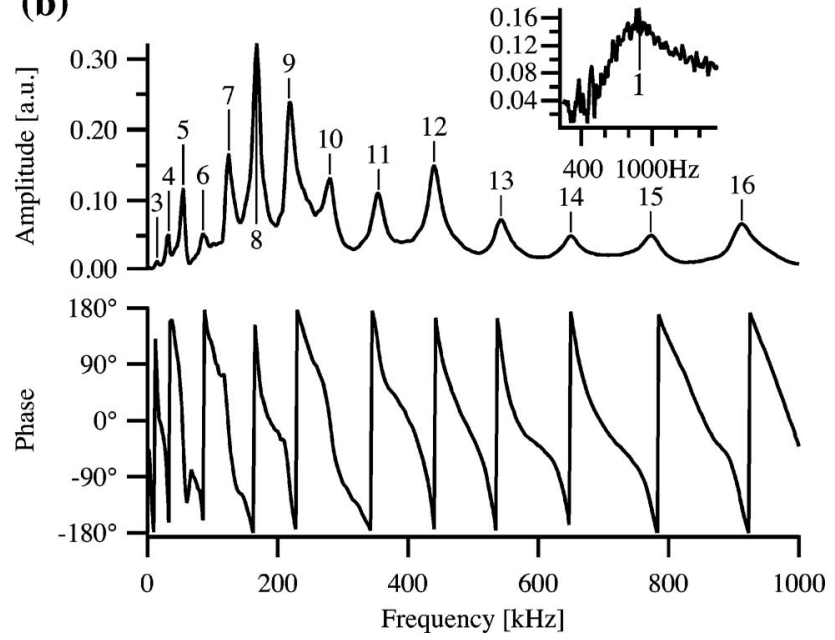

FIG. 1. Recording of resonance spectrum in liquid. (a) Schematic of homemade measurement setup. The cantilevers are excited by a piezoelement beneath the cantilever chip body through a frequency generator. The cantilevers are rotated by $45^{\circ}$ with respect to the liquid cell (dashed line). The read out of the cantilever response signal is performed by a laser deflection system. The frequency analyzer compares the signal from the cantilever with the excitation signal and records amplitude and phase spectra. (b) Complete amplitude and phase spectrum in water; the numbers indicate the mode of cantilever vibration. The amplitude peak of mode 1 is presented as inset. The signal transfer function is discussed in the supplementary material. 


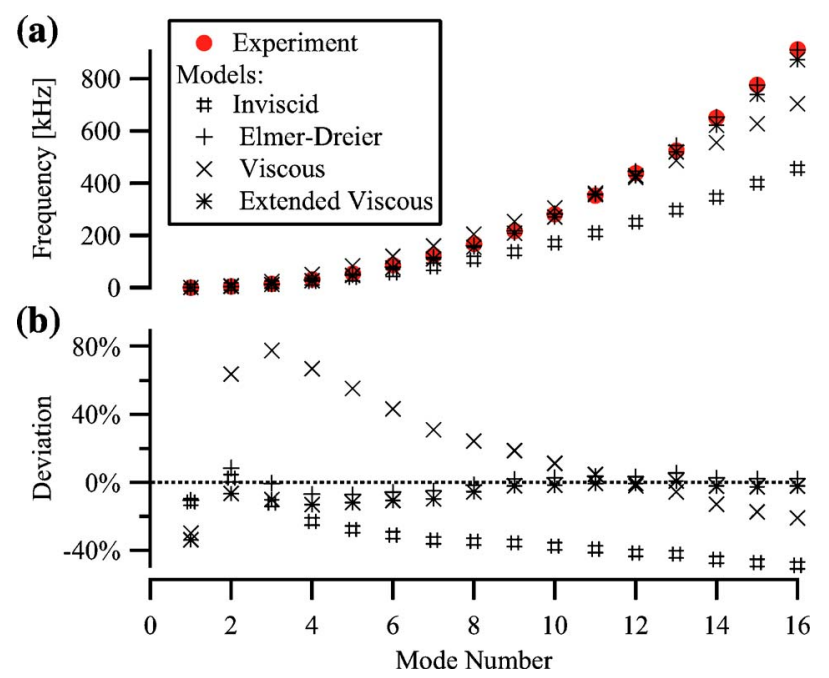

FIG. 2. (Color online) Comparison of experimental eigenfrequencies (red) with the frequencies predicted by different models (black). (a) Absolute frequencies. (b) Relative deviation of the models from the experimental values. Average deviations are 32\% (Inviscid model), 4.4\% (Elmer-Dreier), $30 \%$ (viscous), and $7.1 \%$ (extended viscous model). Note that the determination of the eigenfrequencies at modes 1 and 2 is difficult due to the high damping and the signal transfer function resulting in relative high experimental errors.

eigenfrequencies increased, while the quality factors were linearly spaced. Note that due to the low quality factor at mode 1 the relative error for the eigenfrequency determination is high.

For a microcantilever vibrating in fluid, there is a strong coupling between the fluid and the cantilever resulting in an inertial loading of the beam (also called virtual mass) which cantilevers have to displace owing to the density and viscosity of the surrounding medium. Therefore, the resonance frequency values in fluid are lower compared to those in vacuum. Furthermore, there is strong damping of the vibration in liquid leading to low quality factors. The motion of the fluid is complex and the modal analysis is difficult. Computational fluid dynamic models with sophisticated numeri-

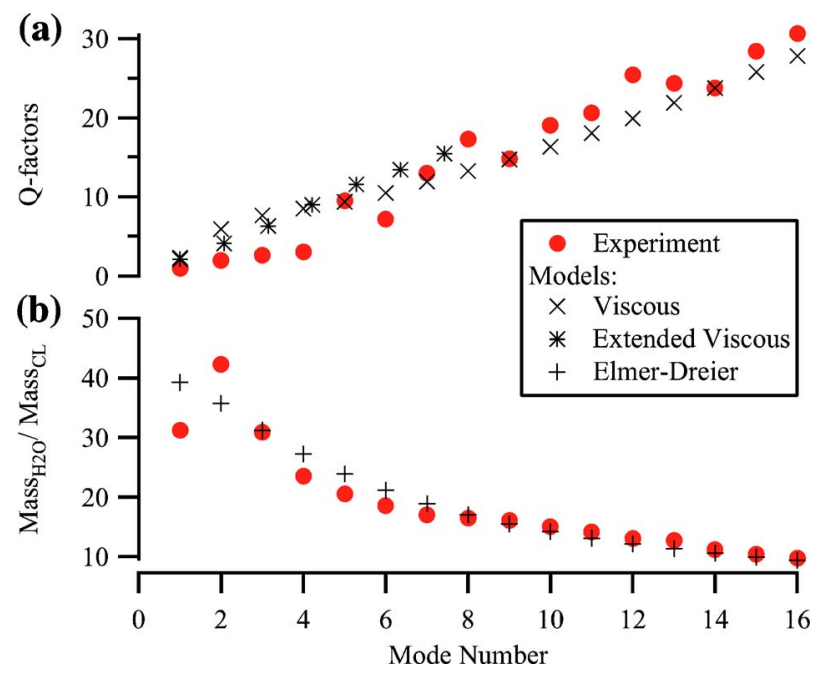

FIG. 3. (Color online) The measured quality factors and virtual mass are compared with model predictions. (a) Quality factors from the amplitude spectra [Fig. 1(b)] compared with the viscous and the extended viscous model. (b) Ratio between the virtual mass and the cantilever mass. Experimental values from the measured eigenfrequency compared with the best matching model from Fig. 2 (Elmer-Dreier model). more than 30\% and underestimated the eigenfrequencies. It
Downloaded 03 Dec 2008 to 131.152.109.224. Redistribution subject to AIP license or copyright; see http://apl.aip.org/apl/copyright.jsp cal techniques considering a three-dimensional (3D) fluid motion around the cantilever have been reported. ${ }^{10-12}$ In our study, we used analytical expressions from different models which can be readily implemented and compared with experimental data. Models considered are the inviscid model from Chu, ${ }^{13}$ the Elmer-Dreier model ${ }^{14}$ for which analytical formulas were derived elsewhere, ${ }^{15}$ Sader's viscous model, ${ }^{16}$ and Sader's extended viscous model for an arbitrary mode number which accounts for the 3D nature of the flow field around the cantilever beam. ${ }^{17}$ The general transcendental expression given by all the models for the eigenfrequency of a vibrating cantilever at an arbitrary mode $n$ in a medium accounting for added apparent mass is ${ }^{16}$

$$
f_{R, n}=\frac{\alpha_{n}^{2}}{2 \pi} \sqrt{\frac{E I}{l^{3} m_{c}\left[1+\frac{\pi \rho b}{4 \rho_{c} h} \Gamma(f)\right]}},
$$

where $E$ is the elastic modulus of the material, $\rho$ is the density of the liquid, $\rho_{c}$ is the density of the cantilever, $l$ its length, $b$ its width, $h$ its thickness, $m_{c}$ its mass, and $I$ $=b h^{3} / 12$ is its moment of inertia. The constants $\alpha_{1}=1.875$, $\alpha_{2}=4.694, \alpha_{3}=7.854, \alpha_{4}=11.0, \alpha_{5} \ldots n=\pi(n-0.5)$ are solutions of the $1+\cos \alpha_{n} \cos \alpha h_{n}=0 .{ }^{18} \Gamma(f)$ is the normalized hydrodynamic load termed as "hydrodynamic function." It is a dimensionless function. Different models considered different approaches for the $\Gamma$ function. Equation (1) corresponds to the frequency where the response of the cantilever is shifted by $90^{\circ}$ compared to the driving force (eigenfrequency obtained from phase). Note that the frequency corresponding to the amplitude peak (peakfrequency) is shifted toward lower value compared to eigenfrequency due to the damping effect.

The thickness of the cantilevers measured by scanning electron microscopy was $0.817 \pm 0.02 \mu \mathrm{m}$. The spring constant value calculated from eigenfrequency of $4.5 \mathrm{kHz}$ in air was $k=0.018 \mathrm{~N} / \mathrm{m}$. The Reynolds numbers determined by $R_{e}=2 \pi \rho f_{\text {wat }} b^{2} / 4 \eta$ (Ref. 16) at various resonance modes ranged between 14 and 14570. The density and viscosity values of nanopure water used were $997.8 \mathrm{~kg} / \mathrm{m}^{3}$ and $9.772 \times 10^{-4} \mathrm{~kg} / \mathrm{m}^{-1} \mathrm{~s}^{-1}$, respectively.

The predicted eigenfrequencies by the theoretical models (black) are shown in Fig. 2(a) besides the experimental data (red). For more detailed comparison, the relative deviation of frequency values obtained from all the models compared to experimentally obtained eigenfrequencies is shown in Fig. 2(b). Note that for mode 1 all model predictions are within the measurement error of the eigenfrequency. Among all the models, the Elmer-Dreier model matched best but also the extended viscous model is in good agreement. The difference between these two models is within the error of the thickness determination of the cantilever (except for mode 1). The good agreement of the Elmer-Dreier model, which is designed for higher modes, is also due to the usage of wide cantilevers reaching inviscid Reynolds numbers at relatively low frequencies. This is in agreement with the validity of the theory for mode number $\geqslant 4$ above which the effect of viscosity was neglected ${ }^{14}$ and was also predicted by Van Eysden and Sader. ${ }^{15}$ The large deviation in the estimation of frequency by viscous model is due to its validity only for low Reynolds number of the order $O(1)$. Moreover it is derived only for fundamental mode. ${ }^{19}$ The inviscid model deviated 
considers a constant added apparent mass $[\Gamma(f)=1]$ for all modes, which was proved to be not the case for microcantilevers by other models. The extended viscous model derived by Van Eysden and Sader ${ }^{17}$ also predicts the frequencies correctly at higher modes. The fundamental frequency matches with the viscous model and for higher modes it follows the Elmer-Dreier model. This indicates that for the dimensions of the cantilever used in the present study, as it resonates from mode 1 to mode 16 the hydrodynamic conditions change from viscous range to inviscid range. Hence the extended viscous model predicts resonance frequencies both at the lower and higher Reynolds number ranges.

We also compared the predicted quality factors with the experimentally obtained values (Fig. 3). An analytical expression is available for the viscous and the extended viscous models to calculate the quality factors but not for the ElmerDreier model. The calculated values by viscous model matched quite well with measured data as predicted previously. ${ }^{17}$ We used the Elmer-Dreier model to calculate the added apparent mass on the cantilever and its value compared to the cantilever mass (93 ng) is shown in Fig. 3(a). The added apparent mass becomes asymptotically smaller for higher modes. A cantilever has to displace almost 40 times of its mass at fundamental and about 10 times at mode 16. This was predicted in the models by Elmer-Dreier ${ }^{14}$ as well as by Van Eysden and Sader. ${ }^{15}$ We provide here an experimental verification. These mass ratios are also in agreement with the calibration method for the virtual mass published recently (data not shown). ${ }^{9}$

In conclusion, we present here a well resolved resonance spectrum for cantilevers vibrating in water. The resonance frequency values of 16 flexural modes and their corresponding quality factors of up to $1 \mathrm{MHz}$ are measured. We found that the eigenfrequencies estimated by the Elmer-Dreier model fits well at mode 8 and beyond as well as those predicted by Sader's extended viscous model. This experimentally proves that the liquid flow around the cantilever truly becomes inviscid at high frequencies. We like to point out that measuring at higher modes has several benefits. First, the quality factors are much higher (a factor of 30 in the present case) leading to smaller experimental error. Second, the virtual mass changes are smaller and change only slightly at higher modes which improves measurement quality and facilitates the calibration of the system. Third, mass sensitivity is at least a couple of orders better at higher mode frequencies ${ }^{20}$ than at fundamental mode. With the knowledge of the correct mode number, the virtual mass can be clearly discriminated in mass measurement experiments. The results and the validation of theoretical models will help to design and improve data analysis for biological mass adsorption experiments in liquid. ${ }^{9}$

M. Ghatkesar and T. Braun contributed equally to this manuscript. We thank Ute Drechsler and Michel Despont from IBM Zurich Research Laboratory for microfabricating the cantilevers. We also acknowledge Professor John Sader from The University of Melbourne, Australia for fruitful discussions and critical comments. The financial support from SNF (NCCR nanoscale science), Endress Foundation and ELTEM Regio is acknowledged.

${ }^{1}$ J. Zhang, H. P. Lang, F. Huber, A. Bietsch, W. Grange, U. Certa, R. McKendry, H. J. Güntherodt, M. Hegner, and C. Gerber, Nat. Nanotechnol. 1, 214 (2006).

${ }^{2}$ J. Fritz, M. K. Baller, H. P. Lang, H. Rothuizen, P. Vettiger, E. Meyer, H. Güntherodt, C. Gerber, and J. K. Gimzewski, Science 288, 316 (2000).

${ }^{3}$ R. McKendry, J. Zhang, Y. Arntz, T. Strunz, M. Hegner, H. P. Lang, M. K. Baller, U. Certa, E. Meyer, H.-J. Güntherodt, M. Hegner, and Ch. Gerber, Proc. Natl. Acad. Sci. U.S.A. 99, 9783 (2002).

${ }^{4}$ Y. Arntz, J. D. Seelig, H. P. Lang, J. Zhang, P. Hunziker, J. P. Ramseyer, E. Meyer, M. Hegner, and C. Gerber, Nanotechnology 14, 86 (2003).

${ }^{5}$ N. Backmann, C. Zahnd, F. Huber, A. Bietsch, A. Plückthun, H. P. Lang, C. Gerber, and M. Hegner, Proc. Natl. Acad. Sci. U.S.A. 102, 14587 (2005).

${ }^{6}$ N. Nugaeva, K. Y. Gfeller, N. Backmann, M. Duggelin, H. P. Lang, H.-J. Güntherodt, and M. Hegner, Microsc. Microanal. 13, 13 (2007).

${ }^{7}$ R. Berger, E. Delamarche, H. P. Lang, C. Gerber, J. K. Gimzewski, E. Meyer, and H.-J. Güntherodt, Science 276, 2021 (1997).

${ }^{8}$ H.-P. Lang, M. Hegner, and C. Gerber, in Applied Scanning Probe Methods II, edited by B. Bhushan and H. Fuchs (Springer, Heidelberg, 2006).

${ }^{9}$ T. Braun, V. Barwich, M. K. Ghatkesar, A. H. Bredekamp, C. Gerber, M. Hegner, and H. P. Lang, Phys. Rev. E 72, 031907 (2005).

${ }^{10}$ M. R. Paul, M. T. Clark, and M. C. Cross, Nanotechnology 17, 4502 (2006).

${ }^{11}$ A. Maali, C. Hurth, R. Boisgard, C. Jai, T. Cohen-Bouhacina, and J.-P. Aiméa, J. Appl. Phys. 97, 074907 (2005).

${ }^{12}$ S. Basak, A. Raman, and S. V. Garimella, J. Appl. Phys. 99, 114906 (2006).

${ }^{13}$ W. H. Chu, South-West Research Institute Technical Report 2 (1963).

${ }^{14}$ F.-J. Elmer and M. Dreier, J. Appl. Phys. 81, 7709 (1997).

${ }^{15}$ C. A. Van Eysden and J. E. Sader, J. Appl. Phys. 100, 114916 (2006).

${ }^{16}$ J. E. Sader, J. Appl. Phys. 84, 64 (1998).

${ }^{17}$ C. A. Van Eysden and J. E. Sader, J. Appl. Phys. 101, 044908 (2007).

${ }^{18}$ D. Young and R. P. Felgar, "Tables of Characteristic Functions Representing Normal Modes of Vibration of a Beam", University of Texas, Austin, TX, Publication 4913, 1949.

${ }^{19}$ J. W. M. Chon, P. Mulvaney, and J. E. Sader, J. Appl. Phys. 87, 3978 (2000).

${ }^{20}$ S. Dohn, R. Sandberg, W. Svendsen, and A. Boisen, Appl. Phys. Lett. 86, 233501 (2005). 